\title{
Researches on the Image Control Point Database
}

\author{
LI Yan \\ Gansu University of Traditional Chinese Medicine \\ Lanzhou, China, 730000 \\ E-mail: ly@gszy.edu.cn
}

\author{
ZHANG Xiao-he \\ Modern Educational Technology \& Network \\ Management Center \\ Gansu University of Traditional Chinese Medicine \\ Lanzhou, China, 730000 \\ E-mail: zyzxh@gszy.edu.cn
}

\begin{abstract}
Ground control point data is the necessary data in the geometric processing of aerospace image photogrammetry. Firstly, this study compared the storage management manners of the control point data. It analyzed the advantages and disadvantages of the file system mode and database mode. Secondly, this study analyzed the features of the control point image data. Consequently, it proposed the database storage solutions of the image control point data. Then it discussed the query methods of the image control point database. Finally, this study designed a control point image database management and application system. The experiment shows that it is effective way to establish the control point image database.
\end{abstract}

Keywords-control point; image data; attribute data; query condition

\section{INTRODUCTION}

Ground control point plays an irreplaceable role in the geometric processing of aerospace image photogrammetry ${ }^{[1]}$ Both collinear equation method and polynomial corrective method need a certain number of ground control points to calculate transformation matrix for the geometric correction. In the traditional methods, the control points are stored in the form of files. This storage form is convenient in the smallscale operations. But it has the defects of low security and low data repeat efficiency. This study adopted the method that all the control points were stored in database. This method has the merits of high data storage security and high data repeat efficiency.

\section{CONTROL POINT IMAGE DATABASE STORAGE STRUCTURE}

\section{A. Control point image data features}

Control point image data contain both attribute data and image data. The attribute data contain location information. That is the geographic coordinates of the control points. This is the main feature of the control point image. Other data that the control point image stores in the database are some necessary supporting information of the geographic coordinates, such as the used coordinate system, projection method, ellipsoid parameters and etc. The control point image data also contains some necessary supporting information of the control point image, such as sensor types, projection method of sensor imaging, height and width of the image, image pixel resolution and etc. Otherwise, there are also the descriptions of the control points features, such as a control point in a small image block is the field corner or road intersection. The above data all could be used as the query conditions. The storage of the attribute data in the relational database is the same with the storage of the general data. It adopted the Two-dimensional table.

The control point image has its own characteristics. One is that it has a small amount of data than other image database. The size of the image is between 100 pixels $\times 100$ pixels and 200 pixels $\times 200$ pixels. The other is that the images have obvious interpretation feature. These characteristics may be field corner, road intersection, walled corner and etc.

\section{B. Data models used to store image data}

On the basis of the control point image characteristics, ZHANG Jixian ${ }^{[2]}$ proposed that the control point image data were stored in the file system and control point attribute data are store in the database. The advantages of this method arethat it is easy to develop and has rapid access speed ${ }^{[3]}$. Meanwhile, it has the defect of low security. The image data stored in the file system could not participate in the database affairs. So these data could not be backed up or restored. If the image data stored in the file system are damaged, the corresponding control points are unusable. So it is better that both the control point attribute data and image data are stored in the database.

In order to organize and manage unstructured data, relational database designs Binary Large Object (BLOB) data. Almost all of the modern relational databases support the BLOB. The BLOB is a manner that it stores the data in the database in the form of binary stream. Even small desktop database support the unstructured data. In the relational-object database, the object-oriented mechanism can be used to store and manage image data. For the Oracle database, the Oracle $8 \mathrm{i}$ vision is partly support objectoriented method. It supports polymorphism. The Oracle 9i vision begins to support object-oriented inheritance. So the Oracle database can adopt suitable object to organize and manage image data. Some methods can be created based on this object. These methods operate on image data at the database side. Oracle database has offered these objects for the users. For instance, the IMAGE in the multimedia options is one of the data image storage management objects 
based on the object mechanism. Oracle 8. 1. 6 offers the ORDIMGB, ORDIMAGE and ORDIMGF objects to store and manage image data.

When the image is stored by object, the object is used as the data type of the two-dimensional table field. This field is used to store not only image data entity, but also the image data attribute information, such as the length and width of the image, file format, compression format and etc. Meanwhile, the object offers some operating method on the image, such as format conversion, data compression, image cutting and etc.

\section{Image control data storage solutions}

On the basis of the above analysis on the control image data features and image data storage in the database, three control point image data storage principles can be summarized as follows:

First of all, both image data and attribute data of the control point image should be stored in the database. Secondly, the image data of the control point image should be stored by the BLOB form in the relational database. In the relational-object database, the image data of the control point image could be stored in the BLOB form or other suitable objects. Thirdly, it also need to store some auxiliary information besides the necessary information. The corresponding storage solution can be designed according to different needs. If the amount of the stored control points is not so large, the desktop database is enough to store control point image data. Microsoft Access, Foxpro, File2Maker Pro are all applicable. If the amount of the control points is large, such as the nationwide control point image database must choose the large database. SQL Server and Oracle are applicable. The blocked strategy could be adopted when there are many control points.

Location information is important in the control point image. So the control point image data can be divided to several parts according to the location information. These parts are stored in different databases. For instance, the nationwide control point image can be divided according to the provinces or graticule of certain size.

\section{QUERY OF THE IMAGE CONTROL POINT DATABASE}

Query function is an important indicator to measure a database. For the control point image database, the query function is also very important. The original intention of creating a control point image is to quickly provide the control point coordinate information for the operator or the application program.

For the control point image database, one important feature is that its ultimate aim is to provide the location information of the control points stored in the database. The query methods ${ }^{[4,5]}$ of the control point image database should be designed around this target. Meanwhile, another characteristic is that the operator or application program should make clear the approximate location before the geometric correction of the image.
The design of the control point image database query method should make full use of these characteristics. On the basis of the above analysis, two kinds of query methods are designed:

\section{A. Query method based on the control point coordinate}

It is known the approximate location of the under corrected image. Then the location information can be used as conditions to query the control point information in the under corrected image range of the database. The image matching or manual method can be used to obtain the geographic coordinates of the control point image in the database. After that, the location information are transferred to the under corrected image.

\section{B. Query method based on the control point feature}

The control point image in the database has obvious interpretation feature. This feature can be used as conditions to query needed control point image.

1) Text mode

Text mode query is that the feature of the control point image can be used as a field stored in the database table. Then the index is created according to the field. When the query is executed, the text attributes can be regarded as conditions.

\section{2) Content-based method}

Not only does the control point image in the database have obvious interpretation feature, but also the control point of the under corrected image has obvious interpretation feature. So the image feature can be used to query the control point image database. First of all, it chooses the range of the under corrected image control points. A certain feature extraction algorithm is applied to extract features of this little block image. Secondly, the same feature extraction algorithm is used to compute features for the control point image in the database. This step can also finish when the data are read. It can improve the query speed when storing image feature fields to the database tables. On the basis of the abstracted features, the content-based query can be realized by giving a certain threshold finally.

\section{THE APPLICATIONS OF THE IMAGE CONTROL POINT DATABASE}

The aim of the image control point database is to effectively organize and manage control point, conveniently obtain the location information of the control points in a certain image area, and geometrically correct the image. A certain query method can be used to query the needed control point image by some information of the under corrected image, such as the approximate location of the control points, image features and etc. An image matching ${ }^{[6]}$ algorithm is used to give the location information of the control point image to the under corrected image. Then the control point coordinate data are obtained automatically. Finally the geometrical correction is completed. The whole process is shown in fig.1. 


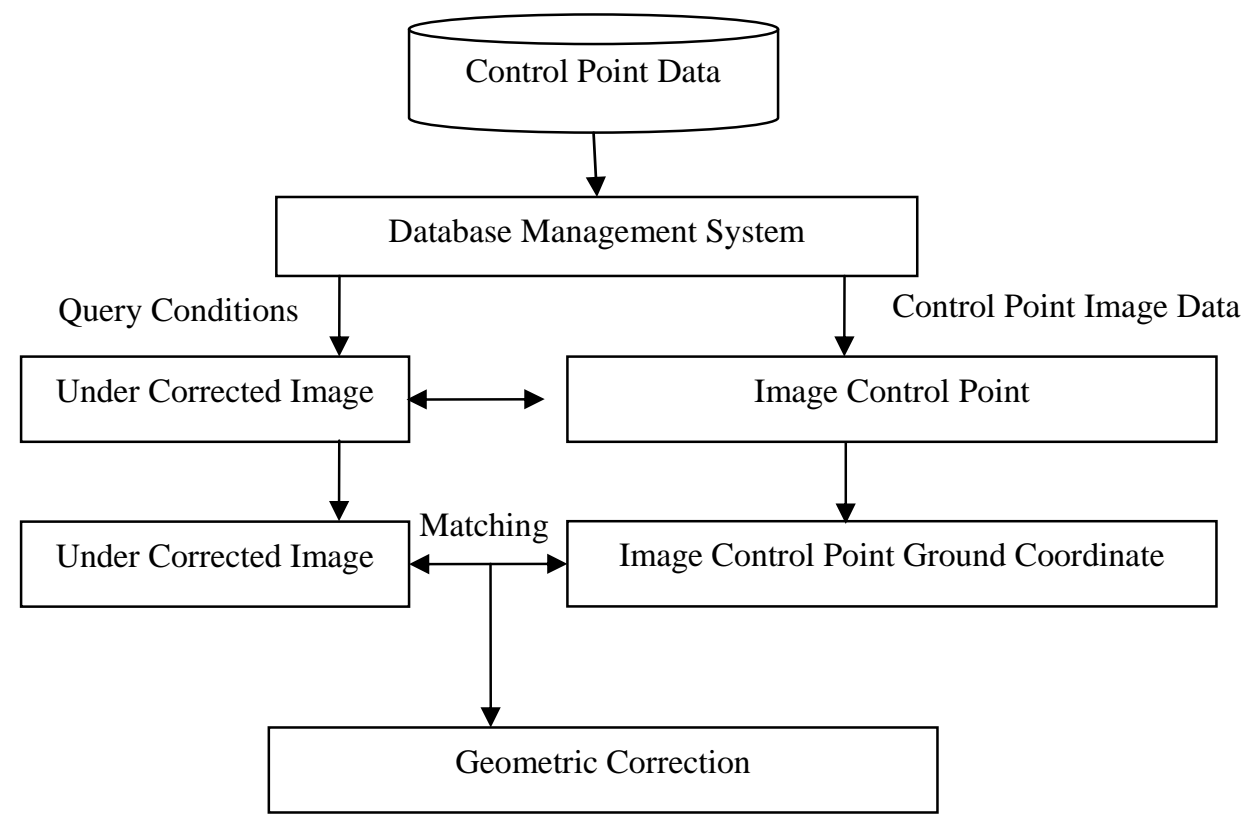

Figure 1. Applications of the image control point database

\section{THE EXPERIMENT OF THE CONTROL POINT IMAGE DATABASE}

The Oracle 8.1.6 was used as the database. The VC++ 6.0 was used as the programming tool. The Ora2cle Call Interface (OCI) produced by the Oracle corp. was used as the interface accessing the Oracle database.

Tab. 1 and tab. 2 are the tables in the database. The data model used to store images was the ORDSYS.IMGB object in the Intermedia options. The attribute data and the image data can be stored in different disks in the computer to reduce the data I/O conflict. Meanwhile, the speed increased.

This paper chose about 300 control point images in two regions. The images are uncompressed. They were stored in the BMP form in the database.

The adopted query method was the integrated approach of the above methods. That was obtaining the control points approximate range in the under corrected image. Then this range was used as a condition. The feature of the under corrected image control point was known. So another condition is the control point feature. The control point images that satisfy the conditions may be more than one. Then the operator visually interpreted which control point image to choose. After that, the ground coordinates of the control points are endowed to the control points in the under corrected image by the matching method. The matching strategy adopted the correlation coefficient method ${ }^{[7]}$ to match the image in the database with the under corrected image.

TABLE I. IMAGE DATA TABLE

\begin{tabular}{llll}
\hline Field & Data types & Constraints & Note \\
Name & & \\
\hline
\end{tabular}

\begin{tabular}{|c|c|c|c|}
\hline ImgNo & VARCHAR(30) & $\begin{array}{l}\text { PRIMAPY } \\
\text { KEY }\end{array}$ & $\begin{array}{l}\text { Control point image } \\
\text { number }\end{array}$ \\
\hline Character & VARCHAR(30) & NOT NULL & $\begin{array}{l}\text { Control piont image } \\
\text { featue description }\end{array}$ \\
\hline Image- X & NUMBER(5. 2) & NOT NULL & $\begin{array}{l}\text { Control piont image } \\
\text { coordinate X }\end{array}$ \\
\hline Image- Y & NUMBER(5. 2) & NOT NULL & $\begin{array}{l}\text { Control piont image } \\
\text { coordinate Y }\end{array}$ \\
\hline ImageData & $\begin{array}{l}\text { ORDSYS. } \\
\text { ORDIMGB }\end{array}$ & NOT NULL & Image data \\
\hline & TABLE II. & \multicolumn{2}{|c|}{ ATTRIBUTE DATA TABLE } \\
\hline Field Name & Data types & Constraints & Note \\
\hline ImgNo & VARCHAR(30) & Foreign key & $\begin{array}{l}\text { Control point } \\
\text { image number }\end{array}$ \\
\hline ImageFileName & VARCHAR(30) & NOT NULL & Image file name \\
\hline Groud- X & NUMBER(11. 3) & NOT NULL & $\begin{array}{l}\text { Control piont } \\
\text { geographic } \\
\text { coordinate X }\end{array}$ \\
\hline Groud- Y & NUMBER(11. 3) & NOT NULL & $\begin{array}{l}\text { Control piont } \\
\text { geographic } \\
\text { coordinate Y }\end{array}$ \\
\hline $\mathrm{Z}$ & NUMBER(6. 2) & NOT NULL & $\begin{array}{l}\text { Control piont } \\
\text { elevation }\end{array}$ \\
\hline
\end{tabular}

\section{CONCLUSION}

The image control point data management by database not only increases the data security, but also reuses the control point data. It realized high efficient management of the control point data. If a certain region in database could store enough control point image data, the geometric correction of the image on this region can reuse the control 
point information. It also could realize the quick input of the control point data.

\section{REFERENCES}

[1] H.J. Buitena, B. van Putten. Quality assessment of remote sensing image registration-analysis and testing of control point residuals. ISPRS Journal of Photogrammetry and Remote Sensing, 1997, 52(2): 57-73.

[2] ZHANG Ji-xian, MA Rui-jin. Control Point Data Base for Graph and Imagery and its Application. Bulletin of Surveying and Mapping, 2000, 1(3): 15-17.

[3] WANG Lian-bei, WU Yun-dong. The Technology of Storing Remote Images Based on Oracle Database. Journal of Institute of Surveying and Mapping, 2002, 19(4): 258-261.
[4] Dimitris Papadias, Jun Zhang, etc. Query processing in spatial network databases. 29th international conference on Very large data bases, 2003, 29: 802-813.

[5] Reynold Cheng, Yuni Xia, etc. Efficient indexing methods for probabilistic threshold queries over uncertain data. The Thirtieth international conference on Very large data bases, 2004, 30: 876-887

[6] Li, W., Leung, H. A maximum likelihood approach for image registration using control point and intensity. Image Processing, 2004, 13(8): 1115 - 1127

[7] Jenn-Hwai Yang, Miin-Shen Yang. A control chart pattern recognition system using a statistical correlation coefficient method. Computers \& Industrial Engineering, 2005, 48(2): 205-221. 